SELF-QUENCHING STREAMERS IN DRIFT TURES

M. Atac and A. V. Tollestrup

Feni National Accelerator Laboratory*

Summary

Self quenching streamers have been observed both optically and electronically. The streamers of 200-300 jm width extend out from the anode wire to 1.5 to $3 \mathrm{~mm}$ at atmospheric pressures. Electronic measurements show that pulses with a rise time of $5 \mathrm{~ns}$ reach $30 \mathrm{mV}$ directly into $50 \Omega$ with a decay time of $40 \mathrm{~ns}$ at a two atmosphere pressure. Details of the experiments and some applications will be discussed.

\title{
Introduction
}

It has been traditionally accepted that gas electron multiplication goes through regions of ionization, proportional, limited proportional, Geiger, and discharge as high voltage of an anode wire is increased in wire counters. 1

This may be the case for same gas mixtures and pressures. Sorne recent works ${ }^{2-6}$ show that unconventional phenomena occur under certain conditions. This paper is about a detailed study of the region above the proportional region which manifests itself as a discontinuous fast transition to a self quenching streamer formation under a continuously applied high voltage. A large current (approaching a milliantpere) results from this transition in less than 100 nanosecond. Optical investigations reveal that narrow streamers, 200-300 $\mu \mathrm{m}$ thick, are formed ortogonal to the anode wire with a length depending on *operated by Universities Research Association under Contract with the United States Department of Energy

Submitted to the proceedings of the IIS International Symposium on Vuclear Radiation Detectors, March 23-26, 1981, Tolyo, Japan. 
applied voltage, the gas mixture, and pressure. The streamers quench thensolves in a well controlled way providing uniform electronic pulses. The results indicate that the width and the height of the streamers decrease as the gas pressure is increased.

\section{Experimental Arrangement}

For the majority of the experiments, extruded aluminum tubes containing anode wires of various thickness were used. Various combinations of argonethane-ethyl alcohol mixture served as the drift tube gas. Some preliminary results were obtained using 50 percent argon, 50 percent carbon dioxide mixture flowing through ethyl alcohol at $0^{\circ} \mathrm{C}$. The results are very similar to that obtained from argon-ethane mixture.

The electronic arrangement was simply either a fast oscilloscope (Tektronix 485) or a pulse height analyzer (LeCroy QVT) for observing pulse shape or detecting charge provided by the self quenching streamer electrons. These devices were directly conrected to the anode wire since there was no need for pulse anplification.

\section{Experimental Results}

A $12 \times 12 \mathrm{~mm}^{2}$ aluminum tube, $100 \mu \mathrm{m}$ diameter gold plated tungsten wire and 49.3 percent $A-49.3$ percent $\mathrm{C}_{2} \mathrm{H}_{6}-1.4$ pexcent $\mathrm{CH}_{3} \mathrm{CH}_{2} \mathrm{OH}$ gas mixture were used for the following results.

Fig. I shows the collected charge as a function of the high voltage starting from the proportional region. Avalanche process slows down as we approach a total charge of $8 \times 10^{6}$ electrons due to space charge saturation. Beyond this value, a discontinuous transition occurs to the self 
quenching streamer mode, and the gain increases much more slowly as the high voltage is increased. For this and the following, a gate width of $300 \mathrm{nsec}$ is used for integrating the charge. Around this transition either the proportional state or the streamer state occurs with no intermediate pulse height observable in between as shown in Fig. 2a and b. These show that the pulse height distribution is Gaussian for cosmic rays as well as the $5.9 \mathrm{keV}$ line of an $\mathrm{Fe}^{55}$ source. Fig. $2 \mathrm{~b}$ indicates that some of the large angle cosmic rays can produce double streamers. For a further investigation of the pulses, a FET probe (Tektronix P6201) with 3 pf capacitance was used between the wire and the soope as shown in Fig. 3. The pulse height was recorded as a function of the load resistor, R. Fig. 4 shows that the pulse height goes up linearly until the FET probe begins saturating. The shape and the magnitude of the pulses are seen in Fig. 5 with several different load resistors. They show that a peak current of $0.8 \mathrm{~mA}$ is provided by the drift tube as a current source. The pulse shape is independent of RC of the circuit indicating that it is governed by the motion of the electrons and positive ions. The pictures were taken around the self quenching streamer transition, thus both the limited proportional pulses and the streamer pulses can be seen in the pictures. As we will show later, electrons and positive ions are produced up to a distance of 2-3 mm depending on the applied voltage.

Collimated $5.9 \mathrm{keV} x$-rays from a $\mathrm{Fe}^{55}$ source of $1 \mathrm{~mm}$ width were used for determining the rate capability of the drift tube for a constant flux. Fig. 6 shows that it is better than $2 \times 10^{3}$ per second per millimeter along the wire. Due to lack of high energy test beams, exact rate capability of the drift tube is not known at this time. 
Efficiency for reaching streamer transition was measured for both 5.9 keV x-rays and minimm ionizing tracks. The results are shown in Figs. 7a and b. It takes considerably higher fields to reach the required space charge for minimm ionizing tracks.

\section{Self Quenching Streamers}

Another drift tube of the type described above was constructed with a conductive transparent window for observing the multiplication process optically around the anode wire. The window is a thin sheet of mylar coated with a very thin film of In-Sn oxide ${ }^{7}$. A two-stage image intensifier RCA video camera (TC1040) was used for detecting photons emitted by the atams involved in the multiplication process. Fig. 8 shows the experimental arrangement. A polaroid camera was used for recording the display on the TV monitor with a shutter speed of $1 / 30$ of a second. Intensity of the source was low enough to record single streamers, double streamers, or no streamers while the pictures were taken randomly. The photographs (Fig. 9) leave no doubt that the fast large electronic pulses are produced by those streamers which start from the wire and extend toward the cathode with a length from $1.5 \mathrm{~mm}$ to about $3 \mathrm{rm}$ depending on the applied voltage and gas mixture. The width of the streamers was measured to be between 200-300 jm using a scale at the same focal distance. Fig. 9d is the result of a multiple exposure of the streamers. They grow in the direction of the initial avalanche. The wire position is indicated at the sides of the pictures. The random bright spots are due to the single photoelectron background of the camera. 
The above data and the further results which will be given suggest the following probable mechanism for the fomation of the self quenching streamers: The avalanche process in the drift tube with $100 \mu$ wire follows the usual exponential behavior in the proportional region as seen in Fig. 1. The rate of growth of the avalanche slows down as the voltage exceeds $2800 \mathrm{~V}$ indicating that space charge saturation is felt by the multiplying electrons. There is a short limited proportional region. Beyond the measured number of $8 \times 10^{6}$ electron-ion pairs in the dense center of the charge clouds, the space-charge field may be so increased that it may negate the applied electric field. As a result, a radiative recombination ${ }^{8}$ of $\mathrm{A}^{+}$and $\mathrm{e}^{-}$may occur, $A^{+}+e^{-} \rightarrow A^{*}+h v$

The energy distribution of these photons is expected to be a continuum since the electron can carry a wide range of energies. Thus, the energy is the sum of the kinetic energy and the binding energy of the electron in that state,

$$
h v=E_{e}+\left(E_{i}-E_{x}\right),
$$

$E_{i}$ and $E_{x}$ are electronic states. Therefore, we may expect some energetic photons reaching outside of the space charge cloud and producing electrons from ethane (first ionizing potential of $\mathrm{C}_{2} \mathrm{H}_{6}$ is $11.78 \mathrm{eV}$ ). A few of these electrons may reach distances of 50-100 $\mathrm{mm}$ to drift back and multiply at the tip of the positive ion cone where the field is the highest. Multiplication should not occur around the cone due to the low field. An artist's view of the phenamena is shown in Fig. 10. Of course, if an electron is created far enough along the wire (possibly $500 \mathrm{fm}$ ) further multiplications oould occur, 
but in this gas mixture such energetic photons (vacuum uv) do not have that chance. Streamer action along the wire happens in Geiger counters due to either low pressure (1/10th of atmospheric pressure) or small amount of quenching gas like 3 percent $\mathrm{C}_{2} \mathrm{H}_{6} \mathrm{Br}$ at atmospheric pressures. Oocurrence of Geiger action (spread of the avalanche along the anode vire) in 3 percent $\mathrm{C}_{2} \mathrm{H}_{6} \mathrm{Br}-97$ percent $\mathrm{A}$, but not in 50 percent $\mathrm{C}_{2} \mathrm{H}_{6}-50$ percent $\mathrm{A}$ is an important evidence that the electrons produced by recombination photons which mediate the self quenching streamers result from ionizing ethane molecules not argon atoms by the process,

$$
\text { hv }+\mathrm{C}_{2} \mathrm{H}_{6} \rightarrow \mathrm{C}_{2} \mathrm{H}_{6}^{+}+\mathrm{e}^{-}
$$

The electric field at the tip of the streamer gets weaker as the length of the streamer gets longer (longer length of positive ion swarm sharing the charge density distribution on the cathode). The streamer photographs taken with the video camera show that they get thinner as they extend further from the anode (Fig. 9).

The following experiments provide more evidence that support the theory above.

\section{Pressure Tests}

For the following experiments, ethyl alcohol was excluded from angonethane gas mixtures to remove one complication in understanding the self quenching streamer mechanism. This required larger size drift tubes to prevent photons reaching the cathode walls and knocking out wall electrons. Fig. Il shows that another phenomenon may occur when the ultraviolet photons reach the cathode walls and produce electrons from the walls. These electrons may drift to the anode wire and continue the multiplication process. 
This appears as a transition from streamer to limited Geiger operation. The pulse width in this mode is about 2 microseconds. The time difference between the rising edge of the streamer pulse and the appearance of the linited Geiger pulse is about 60 nanoseconds which corresponds to an electron drift time from the cathode to the anode wire with $6 \mathrm{~mm}$ separation. The picture was taken for a high voltage value around the transition.

$2 \mathrm{~cm} \times 2 \mathrm{~cm}$ size extruded aluminum tubes provided 400-500 volts wide streamer plateau for anode wires of 50-150 $\mu \mathrm{m}$ diameter.

Fig. 12 shows how the full width of the electronic pulses vary as a function of the pressure for 50 percent $\mathrm{C}_{2} \mathrm{H}_{6}-50$ percent $A$ gas mixture around the streamer transition. It indicates that the streamers get shorter as the pressure is increased due to absorption of recombination photons in shorter distance.

Streamer pulse height was measured (across $50 \Omega$ directly) around transition field values (given in Fig. 13) for various pressures and anode wire thicknesses. It indicates that lower $\mathrm{E} / \mathrm{p}$ values are required with thicker wires. The pulse height reaches an asymptotic value around the $150 \mu m$ wire. An explanation for this is that avalanche may be rolling around the thin vires ${ }^{9}$ thus a substantial fraction of positive ions are shielded from each other. This affects the space charge saturation mentioned earlier thus requiring a higher field for space charge to grow further to make $\mathrm{A}^{+}$and $\mathrm{e}^{-}$ recombination possible. The pulse heights of the limited proportional pulses just below the transition are indicated in Fig. 14. This is another confirmation for reaching space charge saturation with smaller avalanche spread 
around the anode. The avalanche size is further reduced (Fig. 13) by a 1.4 percent admixture of ethyl alcohol to 50 percent $\mathrm{C}_{2} \mathrm{H}_{6}+50$ percent $A$ gas. This is achieved by bubbling the gas through $\mathrm{CH}_{3} \mathrm{CH}_{2} \mathrm{OH}$ at $0^{\circ} \mathrm{C}$.

\section{Light Signals}

Iight emitted from the initial avalanche and the streamer development was further investigated in establishing the time relation between the enission of photons and the wire signal. Fig. 15 shows the experimental arxangement. A drift tube having a transparent window was placed in a dark box with a special photomultiplier tube (Hamamatsu Rl294UX) containing a microchannel plate. Transit time of the phototube was 4.2 nanoseconds with a pulse rise time of 350 picoseconds. A Tektronix 485 oscilloscope was sufficiently accurate to measure time differences between the two signals to 1 nsec in real time. Fig. 16 shows the pulses in coincidence. As seen in the photograph, the wire pulses follow the phototube pulses well up to 100 ns. The wire current continues to flow up to $170 \mathrm{~ns}$, while the phototube current diminishes around $120 \mathrm{~ns}$ indicating that the electrons produced at the tip of the streamer are drifting to the anode wire. Positive going pulses, which are the light pulses, have a considerably wider distribution than the negative going wire pulses. This is due to the time structure of the photons emitted during the sucoessive avalanche formation. Superimposed pulses with such structure widens the pulse height distribution. The number of successive avalanches average around 10. This structure can be seen in Fig. 8. The time relation as a function of the gas pressure is shown in Fig. 17. The time difference between the trailing edges gets smaller as the pressure 
is increased (indicated in Fig. 18) showing that the height of the self quenching streamers is decreased. The minimm height is around $2.5 \mathrm{~mm}$ at one atmosphere pressure, and it goes down to $0.3 \mathrm{~mm}$ at two atmosphere taking a drift time of $200 \mathrm{nsec}$ per centimeter.

\section{Acknowledgment}

The authors would like to express their appreciation to Drs. K. Kondo, A. Menzione, and M. Mishina for useful discussions and to D. Hanssen, M. Hrycyk, J. Urish, and Y. Yasu for their contributions. 


\section{Figure Captions}

Fig. I Measured charge as a function of the high voltage for a gate width of 300 nsec.

Fig. 2 Self quenching streamer pulse height distributions for $5.9 \mathrm{keV}$ $x$-rays and cosmic rays. The second peak in the latter case is due to double streamer formation for large angle cosmic rays.

Fig. 3 The circuit diagram for observing the pulse shapes.

Fig. 4 Pulse height versus the load resistance.

Fig. 5 Streamer pulses with various load resistors.

Fig. 6 Count rate capability of a wire in the streamer mode.

Fig. 7a Streamer transition efficiency for $5.9 \mathrm{keV}$ x-rays.

Fig. Tb Streamer transition efficiency for minimum ionizing hadrons.

Fig. 8 Experimental arrangement for photographing individual streamers.

Fig. 9 The photographs of the individual self quenching streamers. Anode wire position is indicated at the sides of the pictures. Fig. $9 \mathrm{~d}$ is the result of a multiple exposure.

Fig. 10 Artist's description of the self quenching streamer phenorena.

Fig. Il Self quenching streamer and Geiger pulses superimposed.

Fig. 12 Full width of streamer pulses as a function of gas pressure just above the streamer transition threshold.

Fig. 13 Streamer pulse height as functions of pressure and wire diameter just above the streamer threshold.

Fig. I4 Limited proportional pulse height as a function of wire diameter just below the streamer transition.

Fig. 15 Experimental arrangement for measuring timing between the photon and the wire current during the self quenching streamer formation.

Fig. 16 Phototube and the wire pulses are superimposed. Positive going ones are the phototube pulses. Horizontal scale is $50 \mathrm{~ns} /$ division, vertical scale $20 \mathrm{mV} /$ aivision. 
Fig. 27 Full width of the wire and the phototube signals as a function of the gas pressure.

Fig. 18 Drift time and streamer heights as a function of the gas pressure. 


\section{References}

Ref. 1 S. A. Korf, Electron and Nuclear Counters, p. 13.

Ref. 2 C. Grumberg et al., Nucl. Instr. and Meth. 78 (1970) 102.

Ref. 3 S. Brehin et al., Nucl. Instr. and Meth. 123 (1975) 225.

Ref. 4 G. Battistoni et al., Nucl. Instr. and Meth. 164 (1979) 57.

Ref. 5 G. D. Alekseev et al., Nucl. Instr. and Meth. 177 (1980) 385.

Ref. 6 M. Atac, IFEE Trans. Nucl. Sci., Vol. NS-28 (1981) 492.

Ref. 7 M. Atac, Nucl. Instr. and Meth. 176 (1980) $\lambda$.

Ref. 8 D. R. Bates, Atomic and Molecular Processes.

Ref. 9 H. Okuno et al., IEEE Trans. Nucl. Sci., NS-26, No. 1 (1979) 160. 


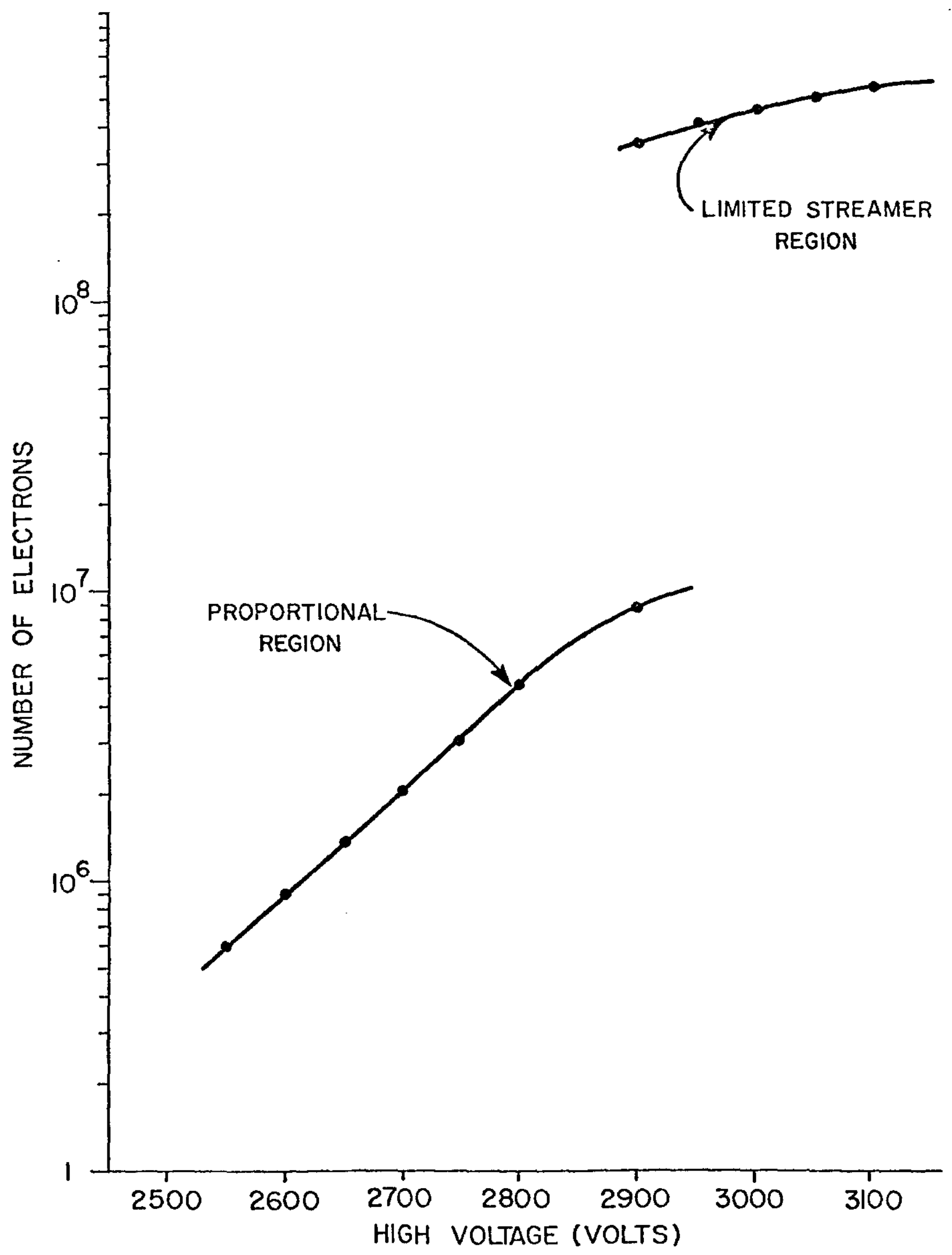

Fig. 1 


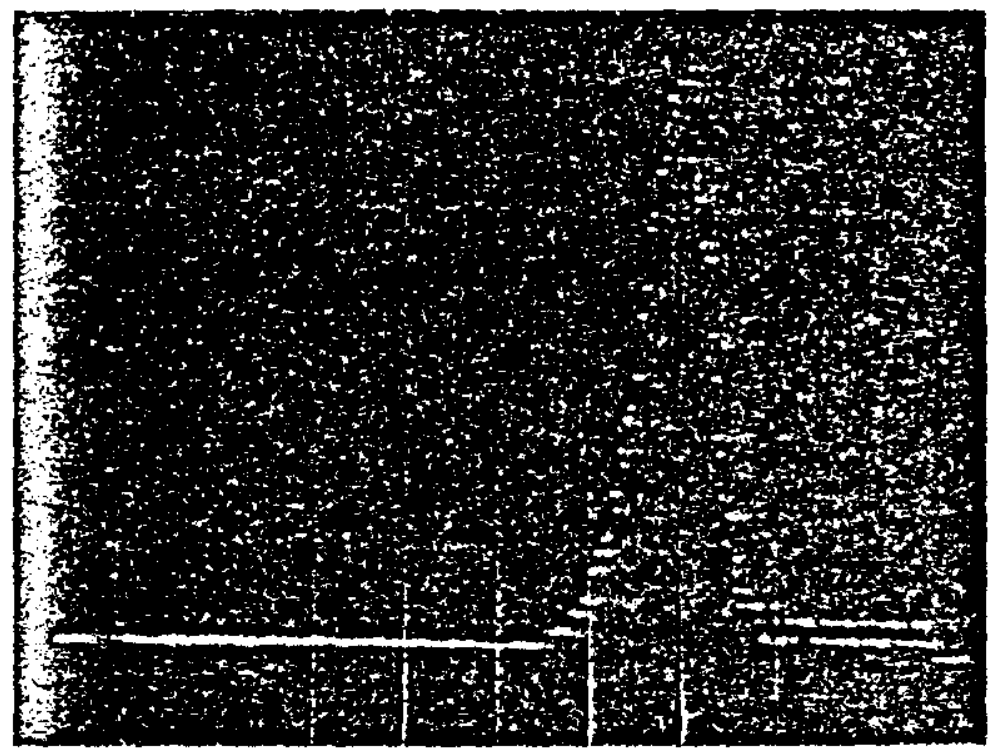

$5.9 \mathrm{keV} F e^{55}$

a.)

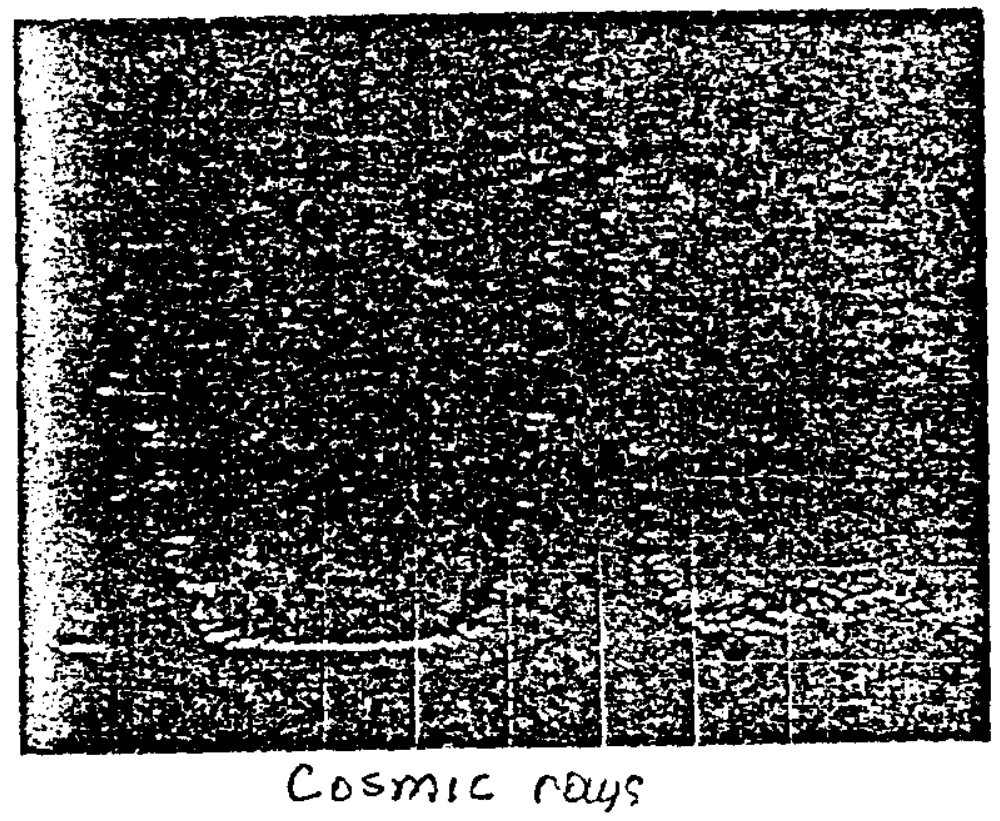

b.

Fig. 2 


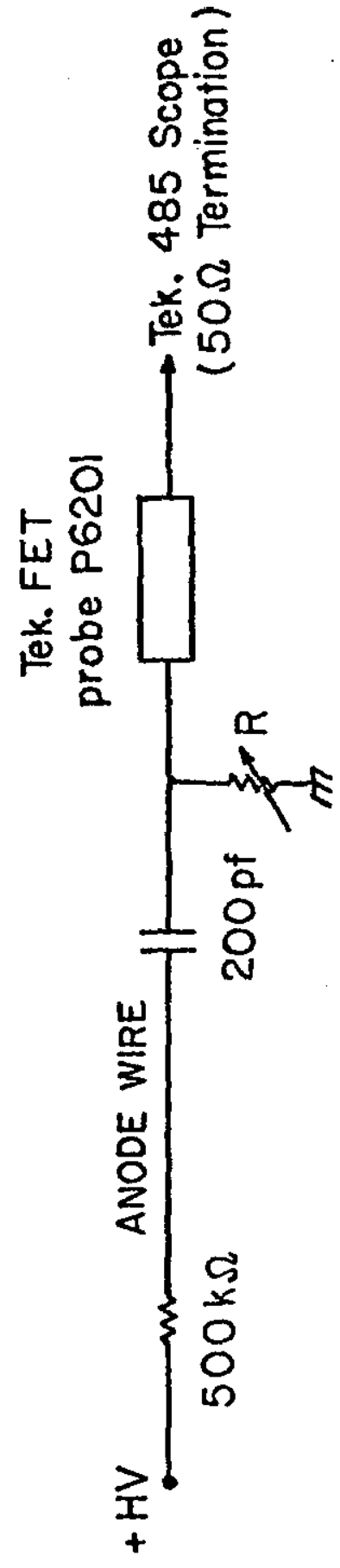

m 


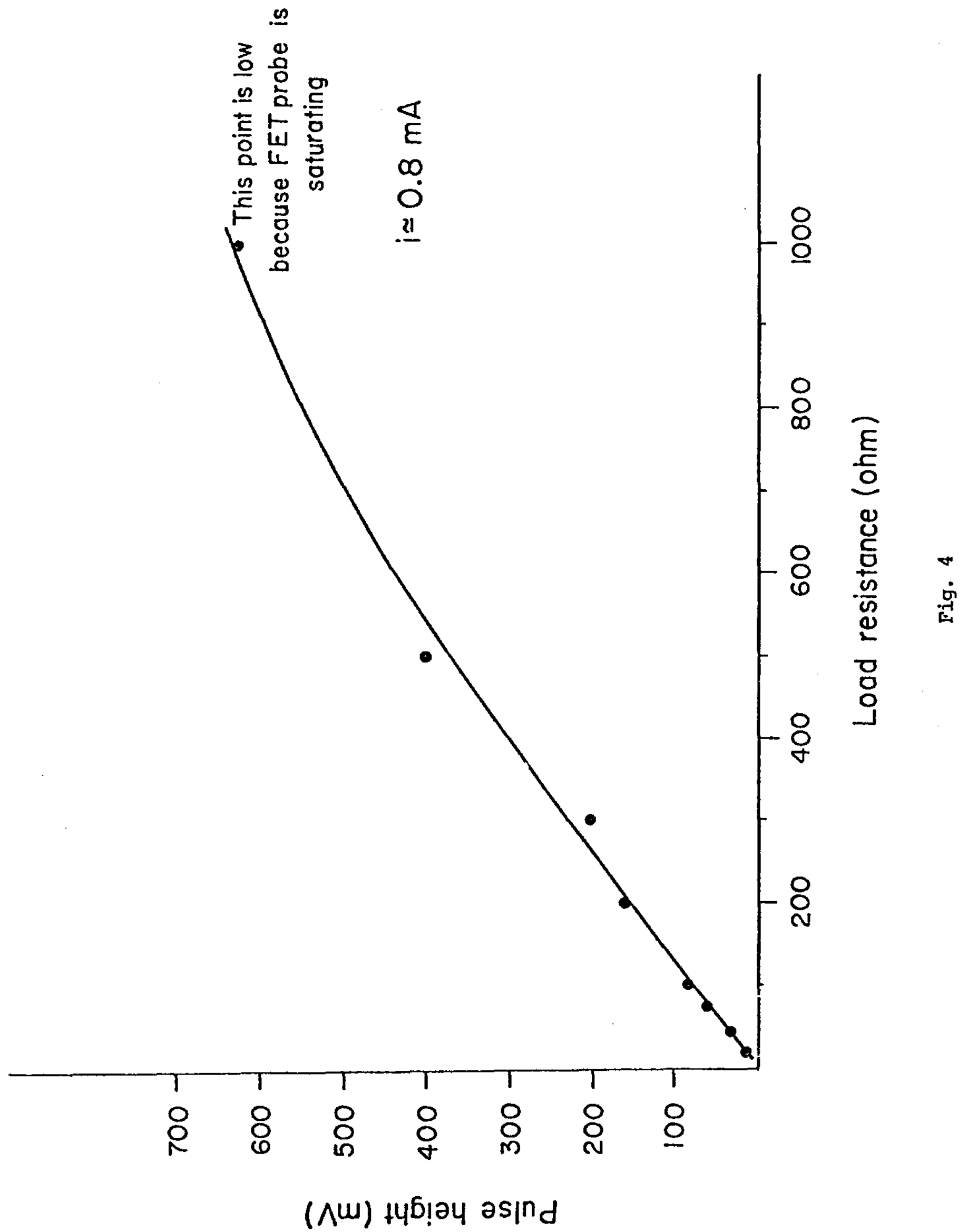




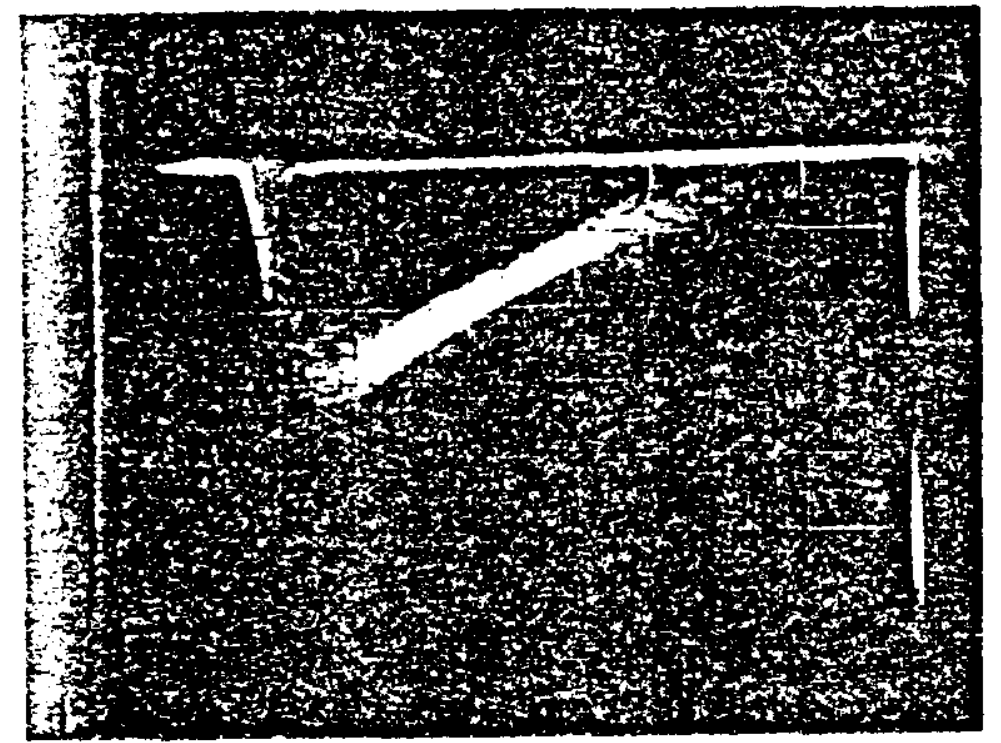

$$
\text { a., } \begin{aligned}
& r=75 \Omega \\
& 20 \mathrm{mv} / \mathrm{div} \\
& 20 \mathrm{~ns} / \mathrm{div}
\end{aligned}
$$

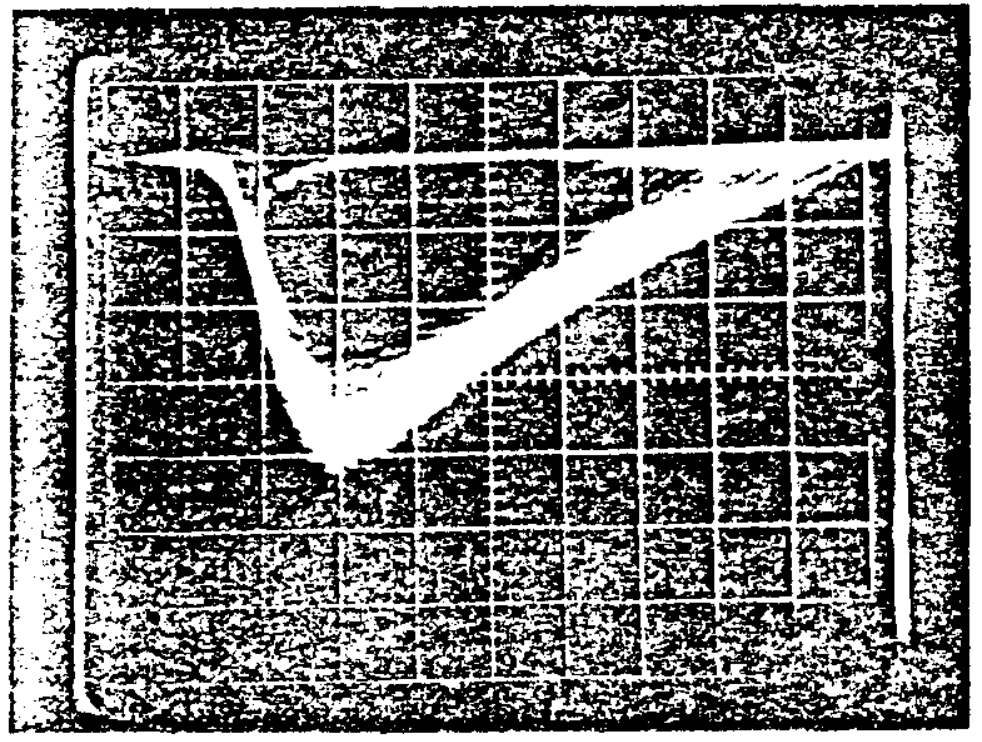

$$
\text { c., } \begin{aligned}
& r=500 \Omega \\
& 100 \mathrm{mV} / \mathrm{div} \\
& 20 \mathrm{~ns} / \mathrm{div}
\end{aligned}
$$

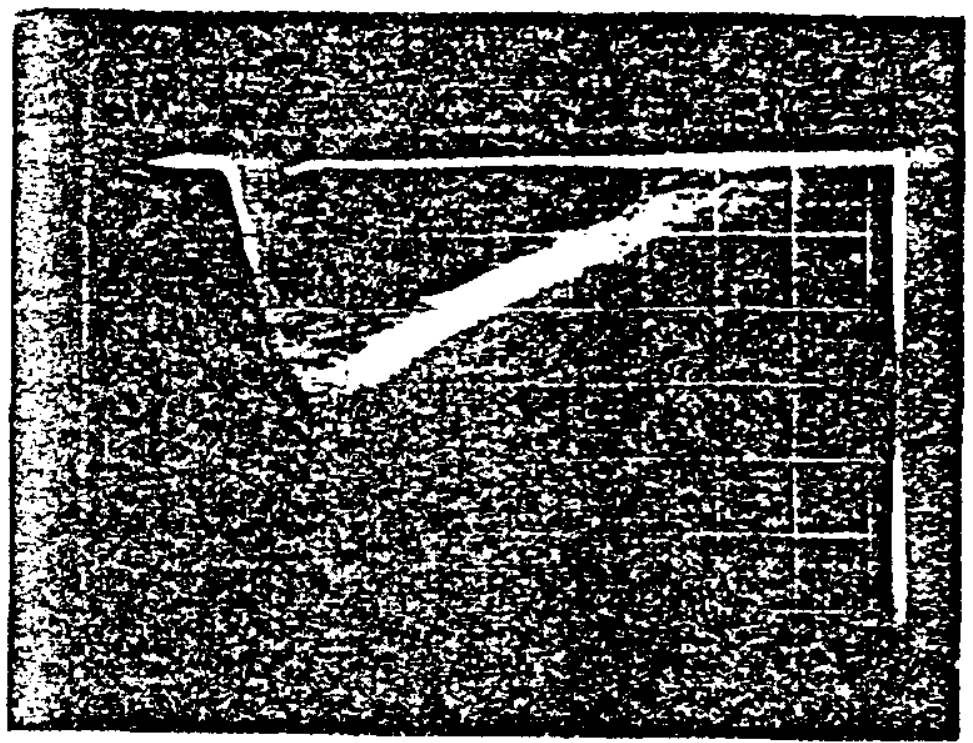

$$
\text { b., } \begin{array}{r}
r=200 \Omega \\
50 \mathrm{mV} / \mathrm{div} \\
20 \mathrm{~ns} / \mathrm{div}
\end{array}
$$

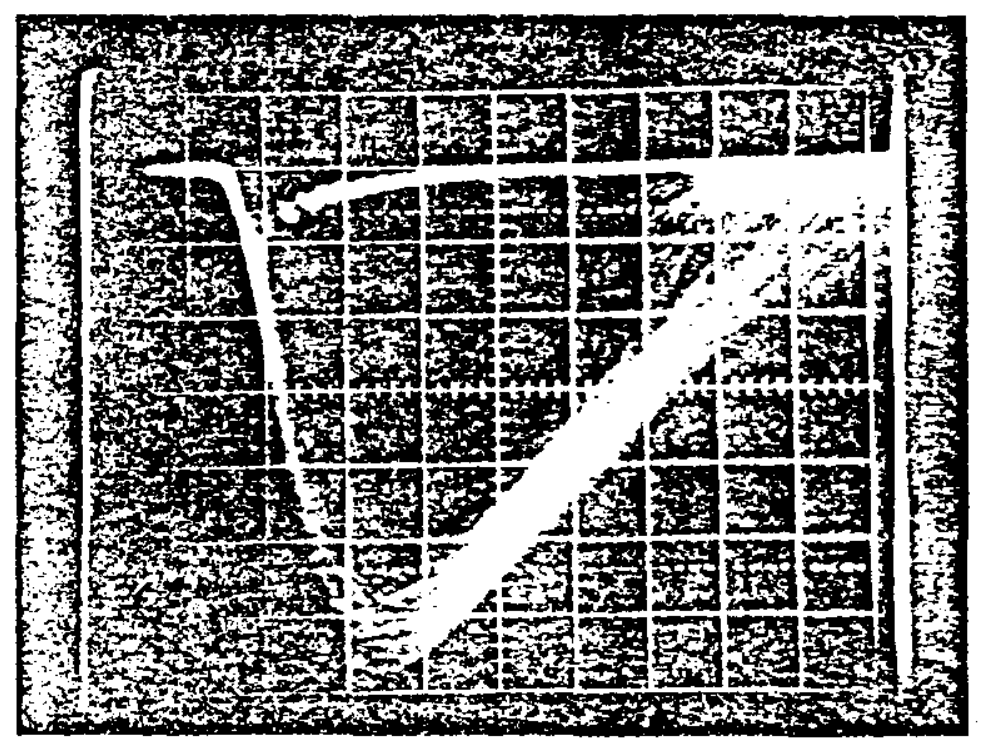

$$
\begin{aligned}
& \text { d., } r=1 \mathrm{k} \Omega \\
& 100 \mathrm{mV} / \mathrm{div} \\
& 20 \mathrm{~ns} / \mathrm{div}
\end{aligned}
$$




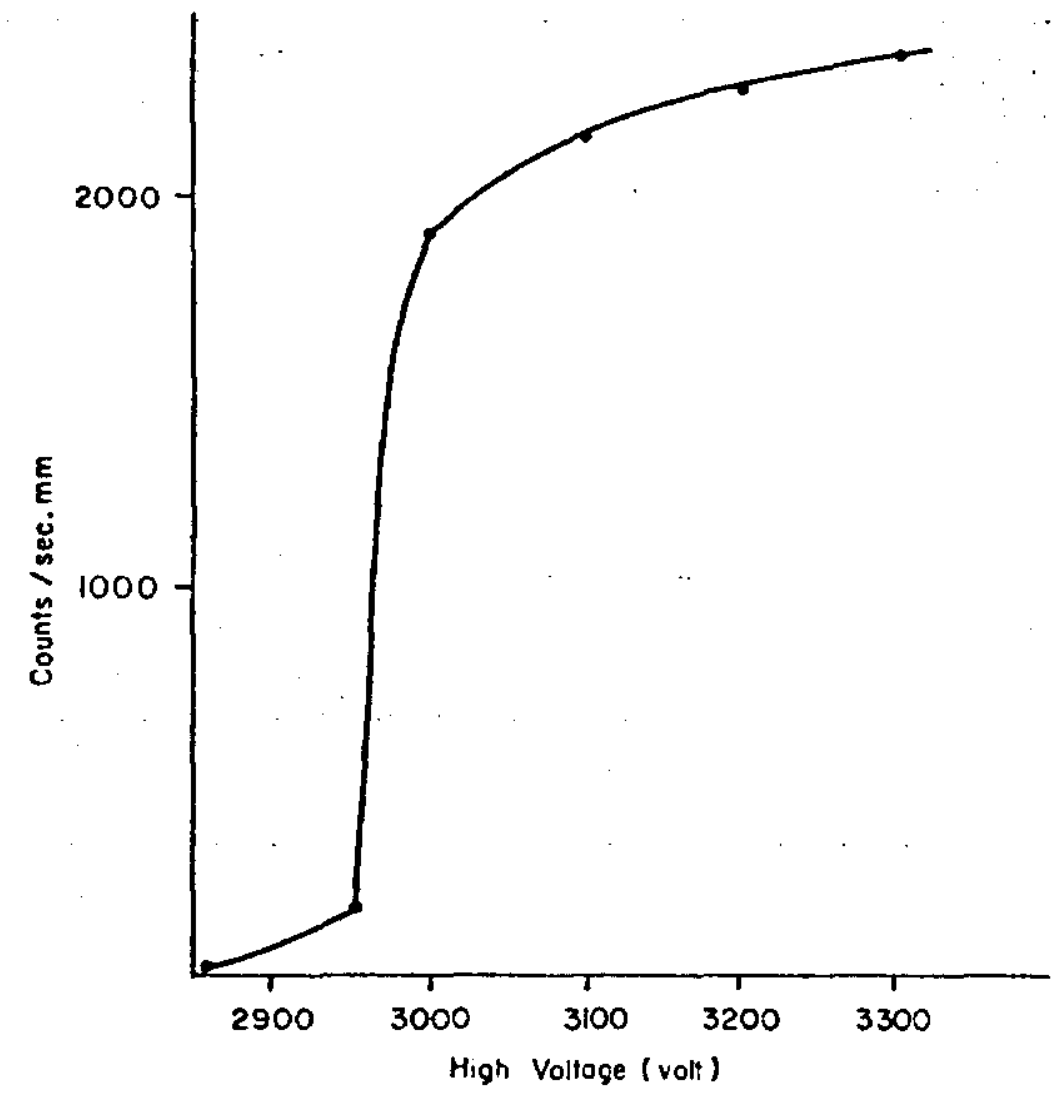

Fig. 6 


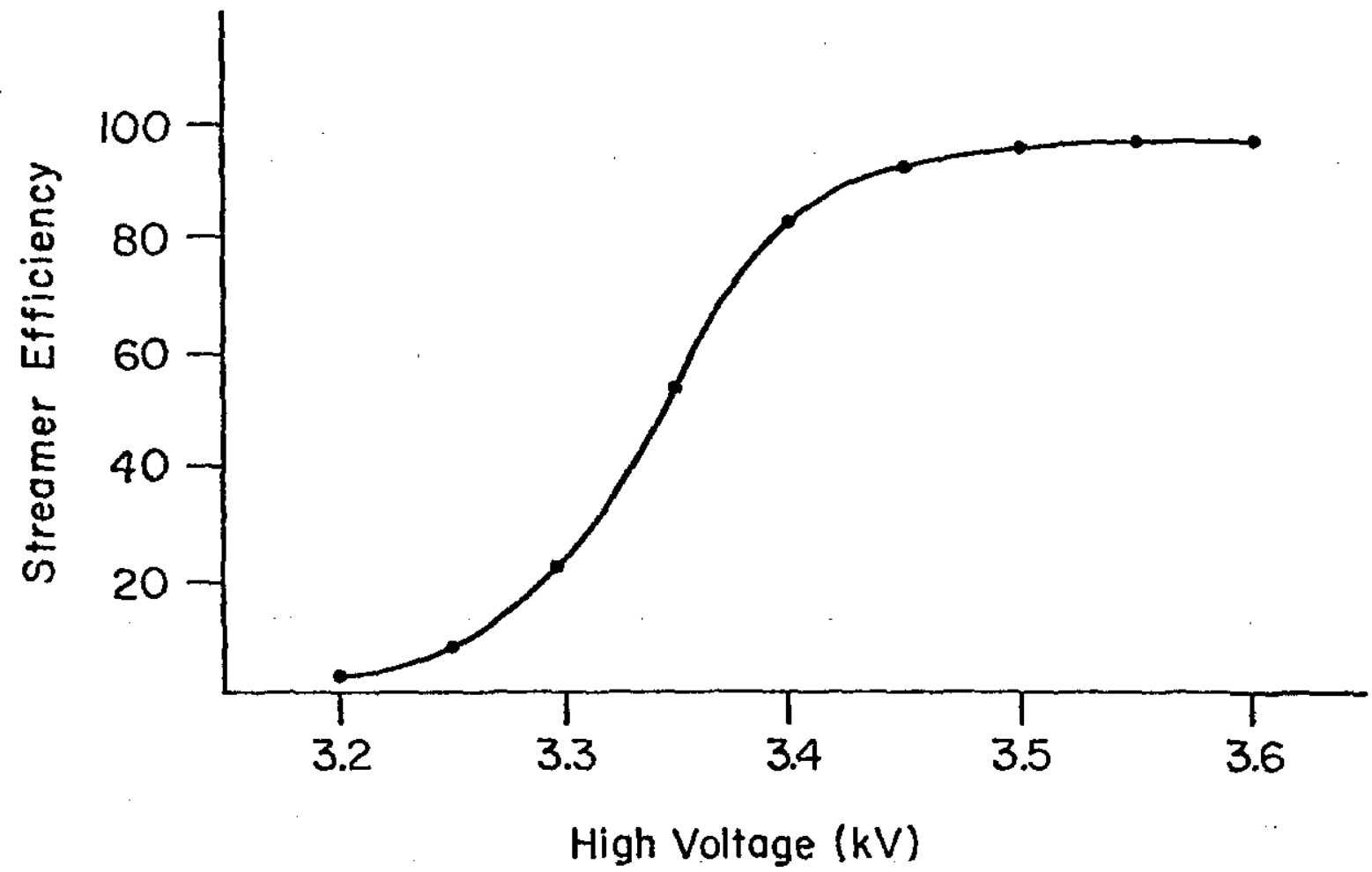

Fig. 7a. 


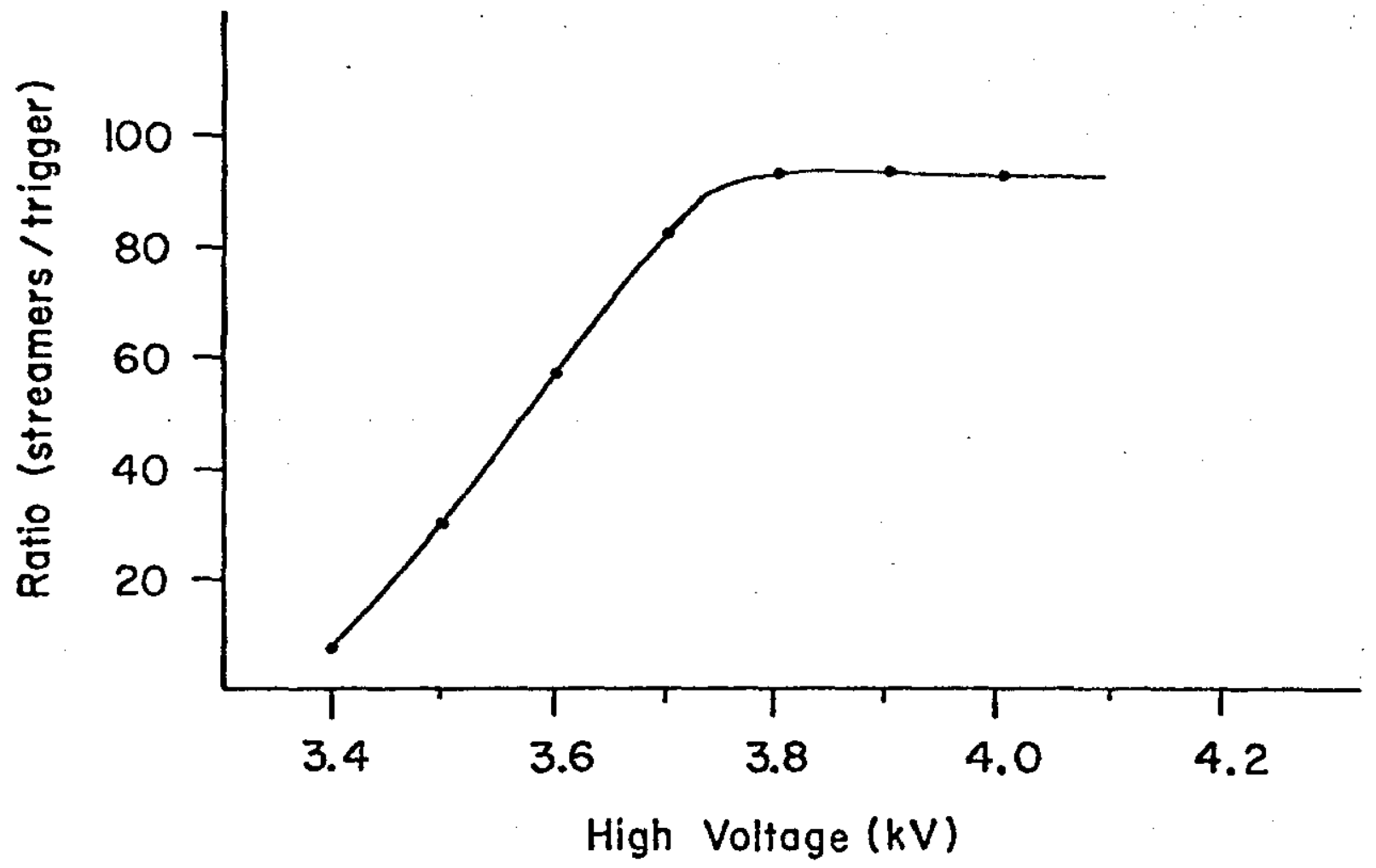

Fig. $7 \mathrm{~b}$. 


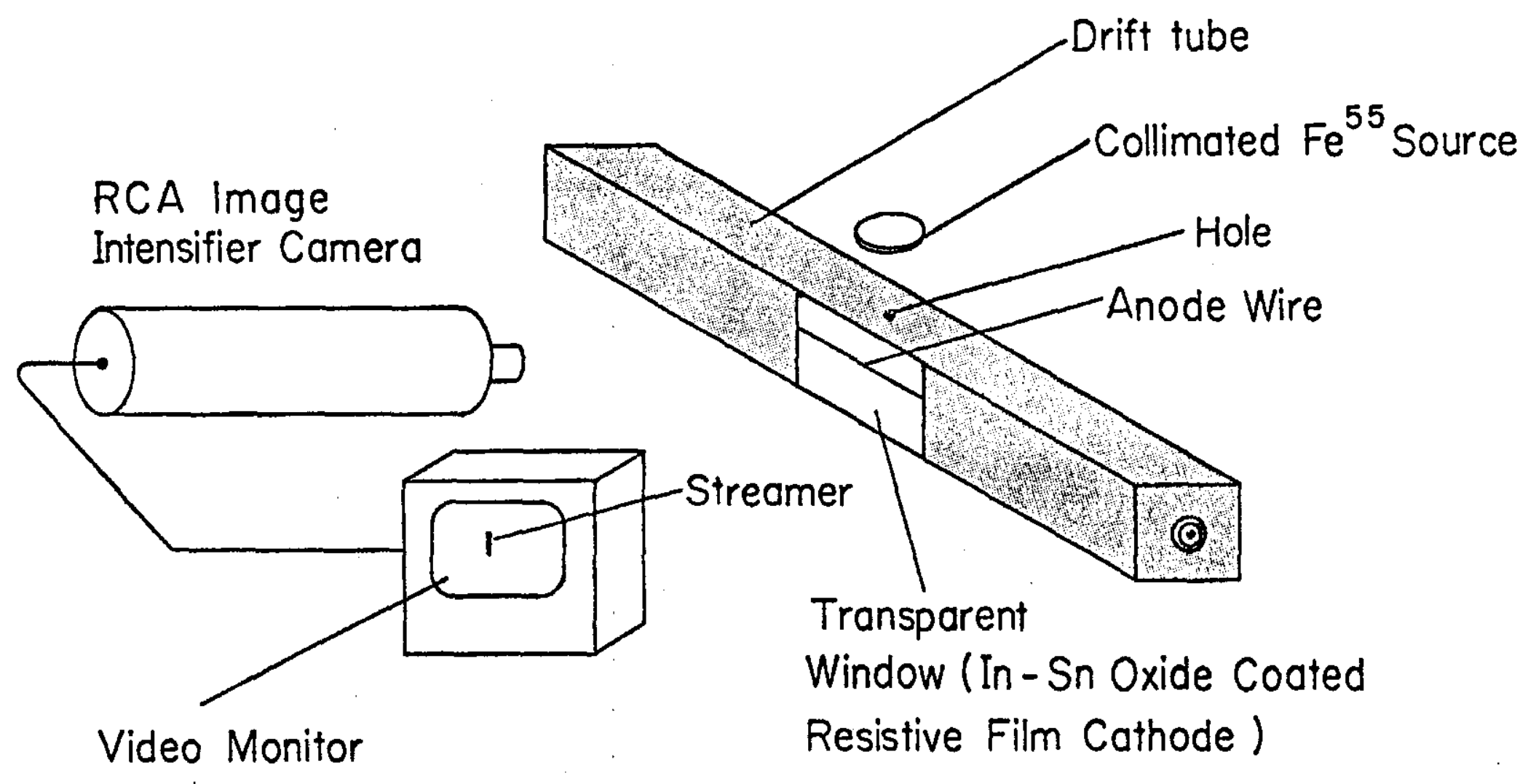

Fig. 8 


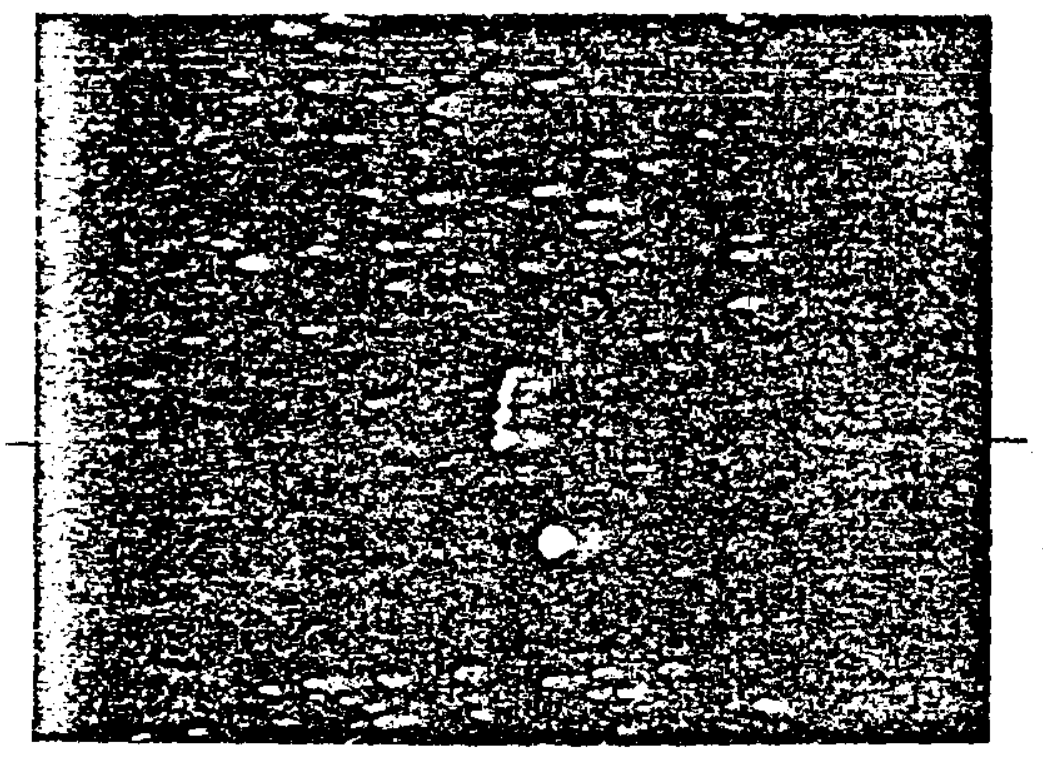

a.

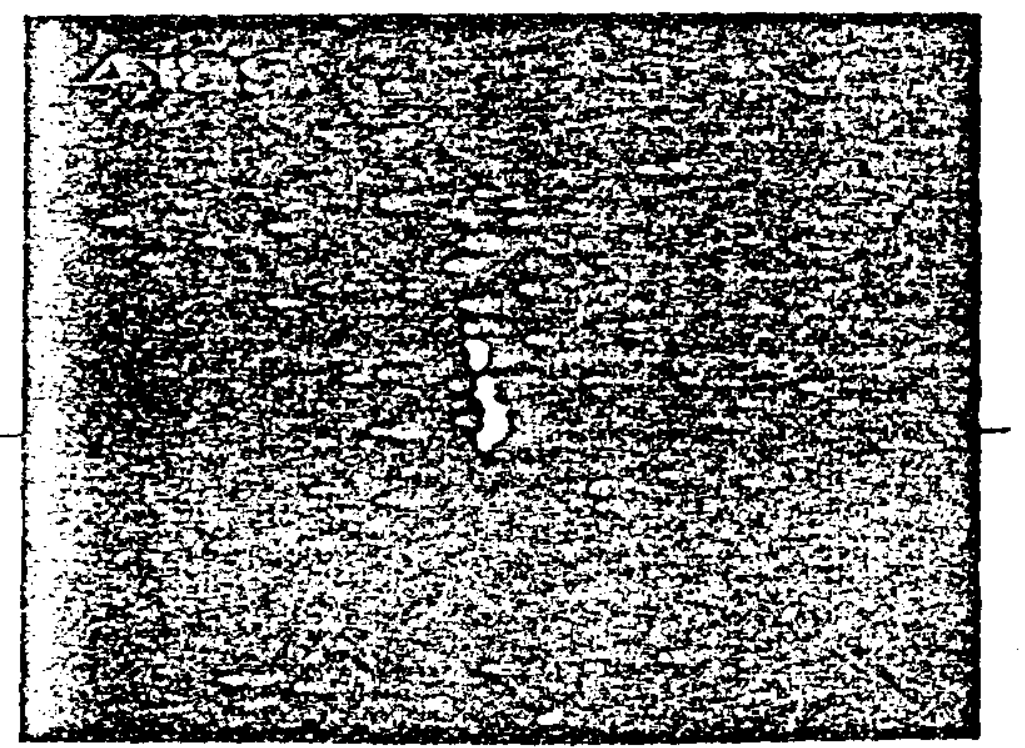

c.

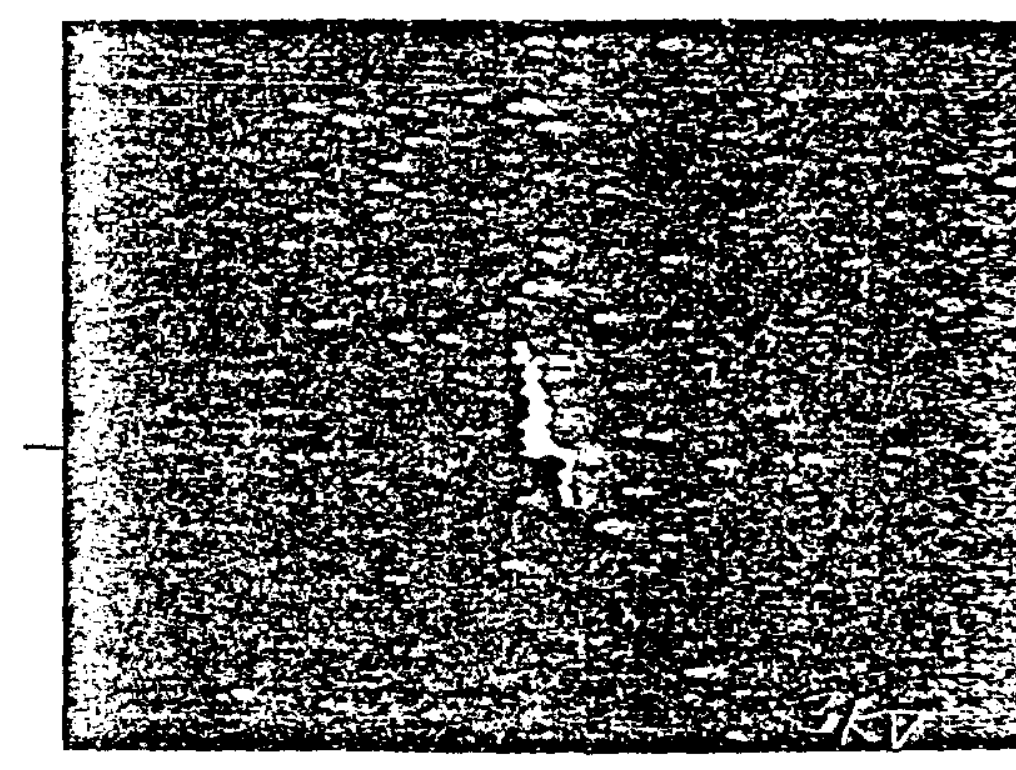

b.

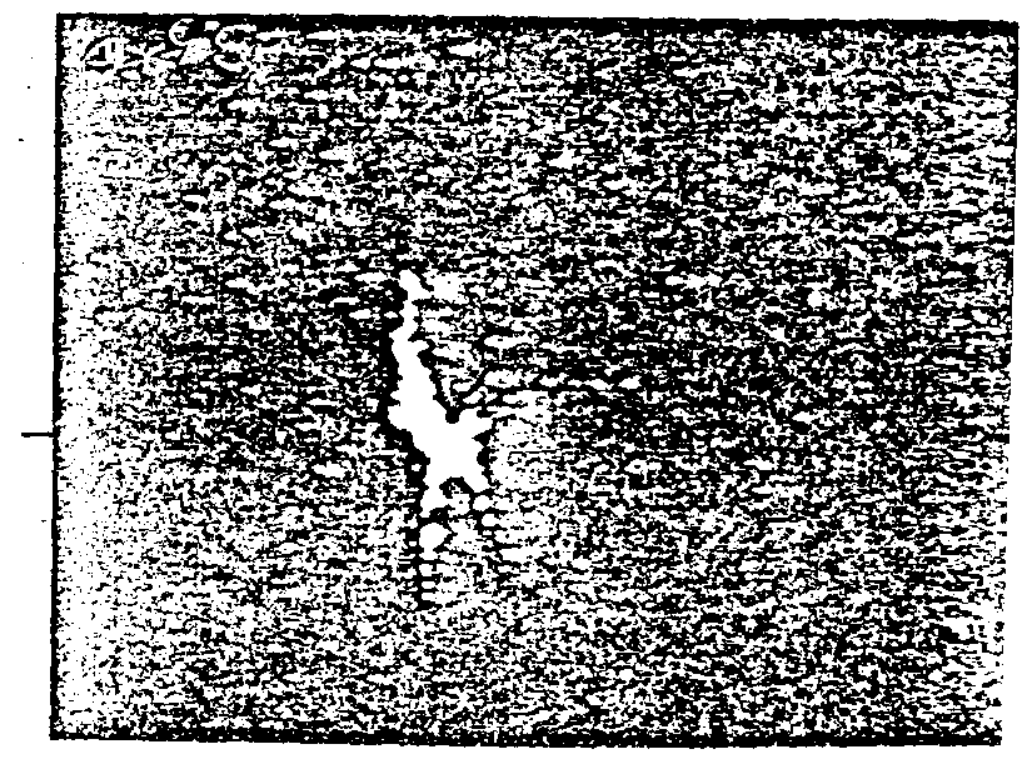

a.

Fig. 9 

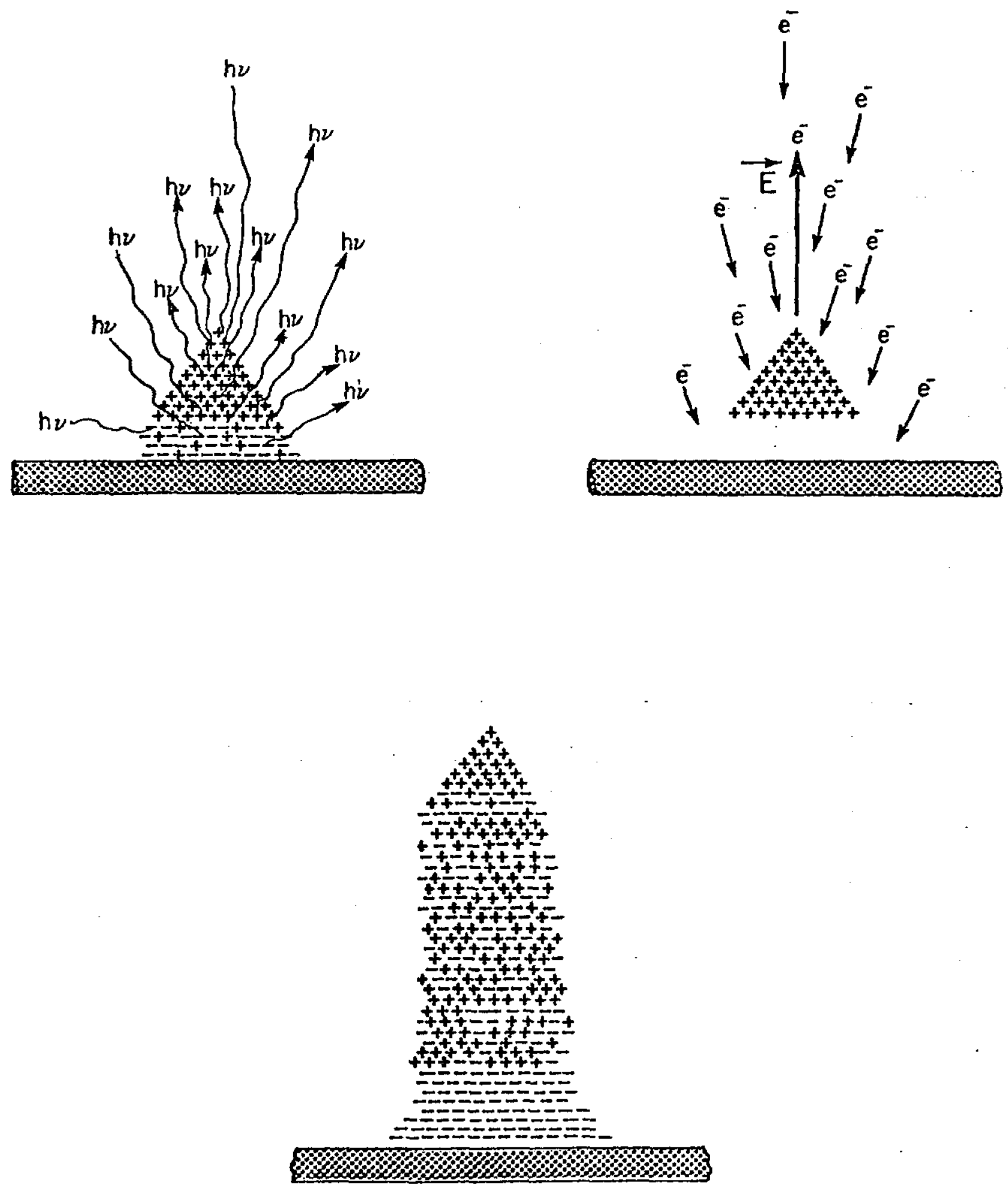

Fig. 10 


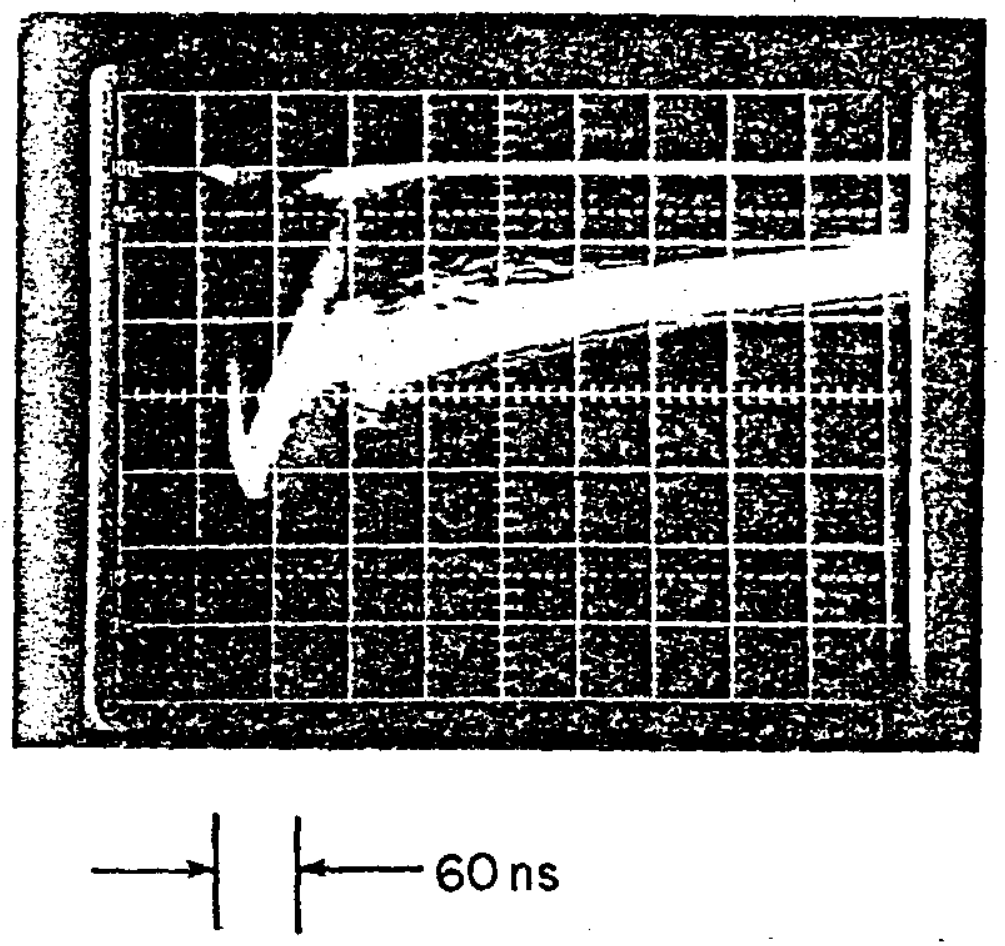

Fig. 11 


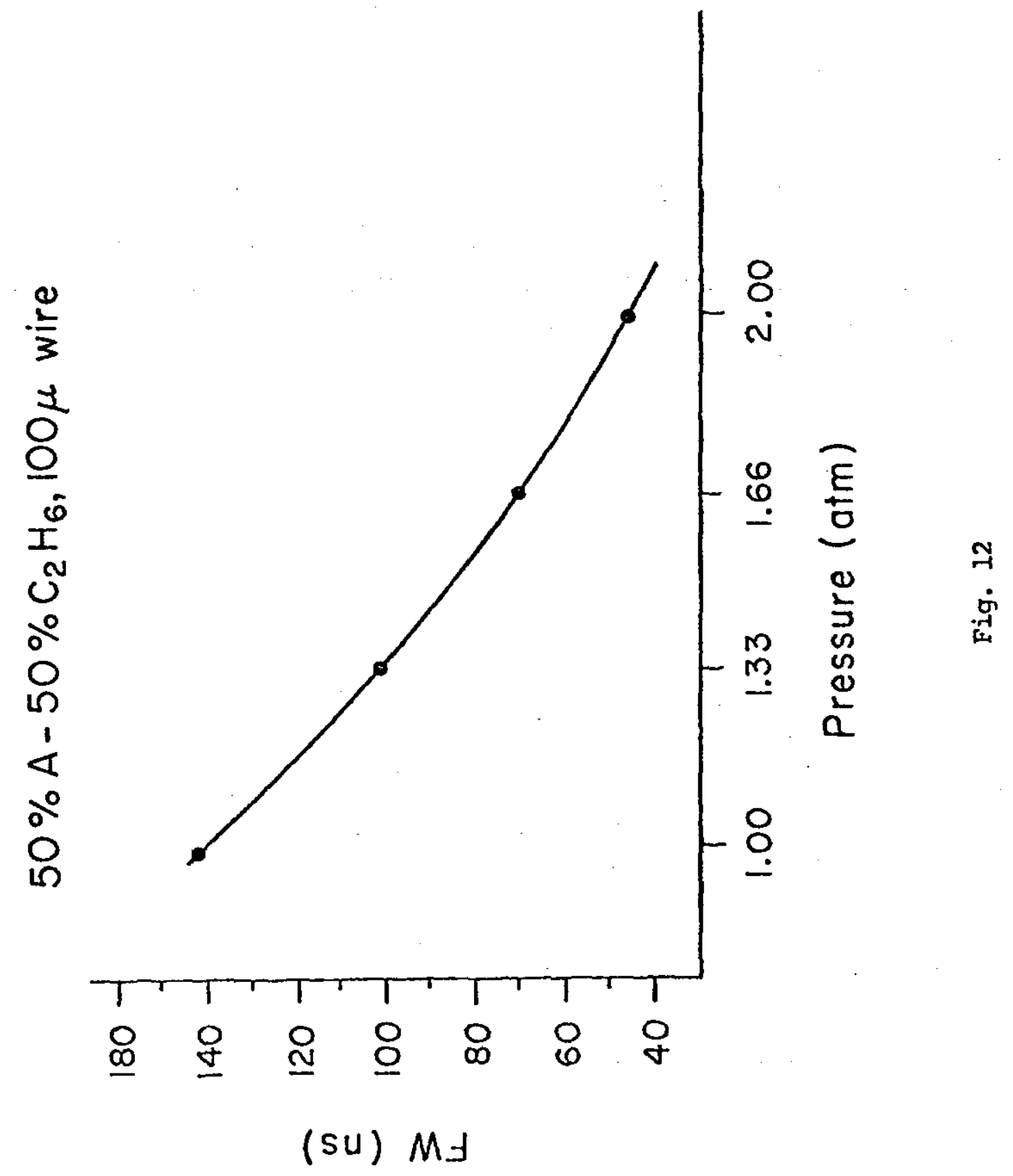




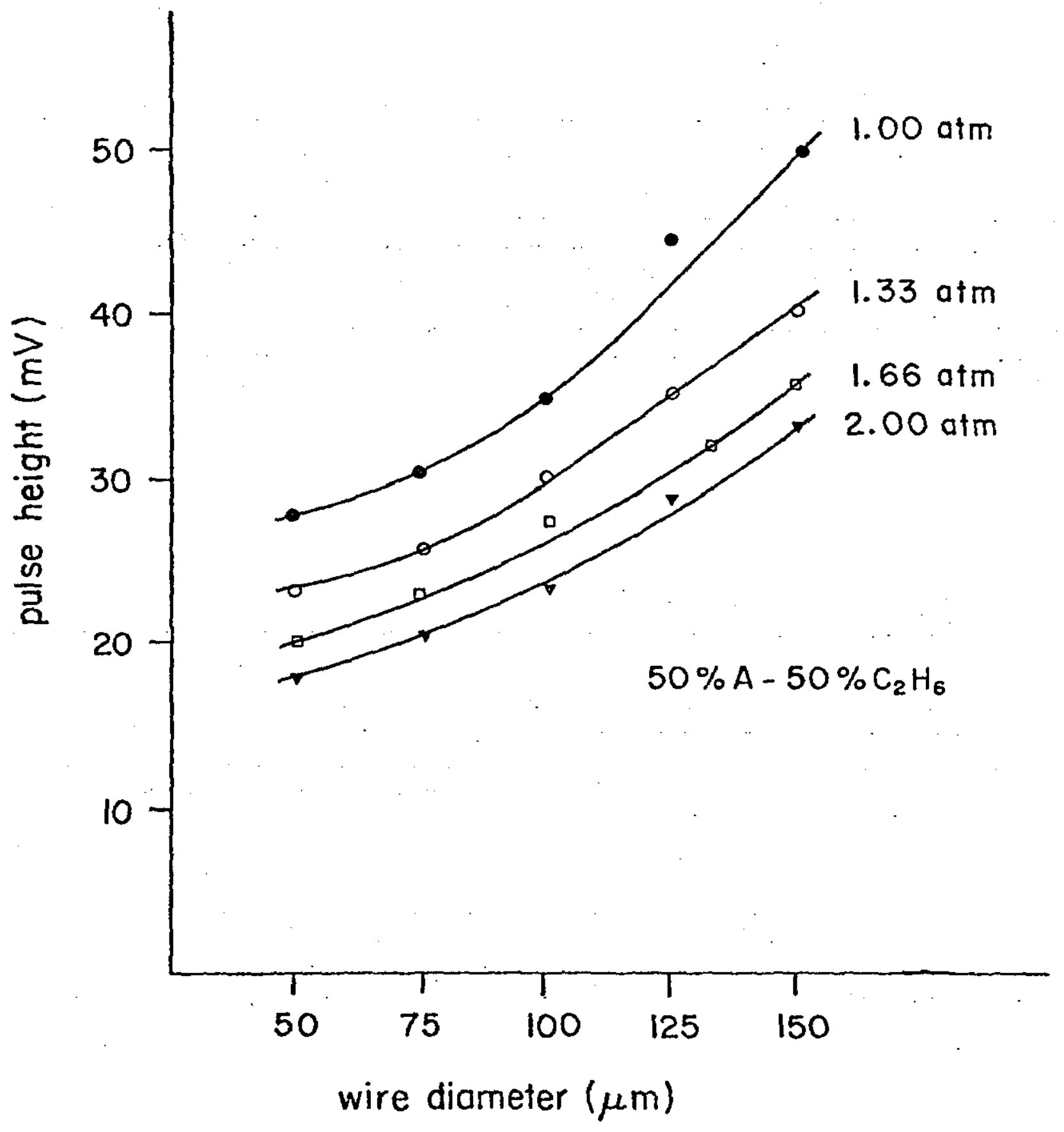

Fig. 13 


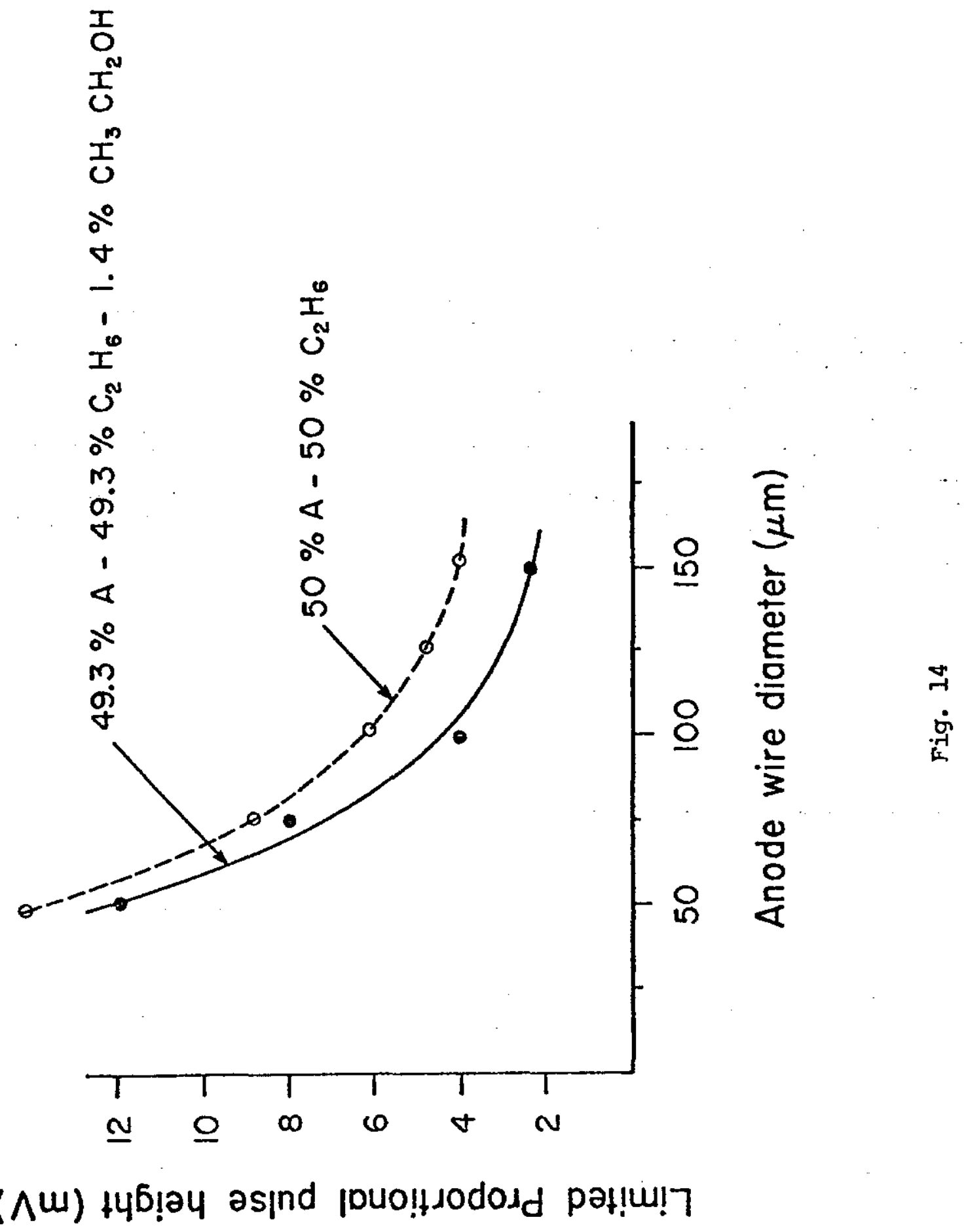




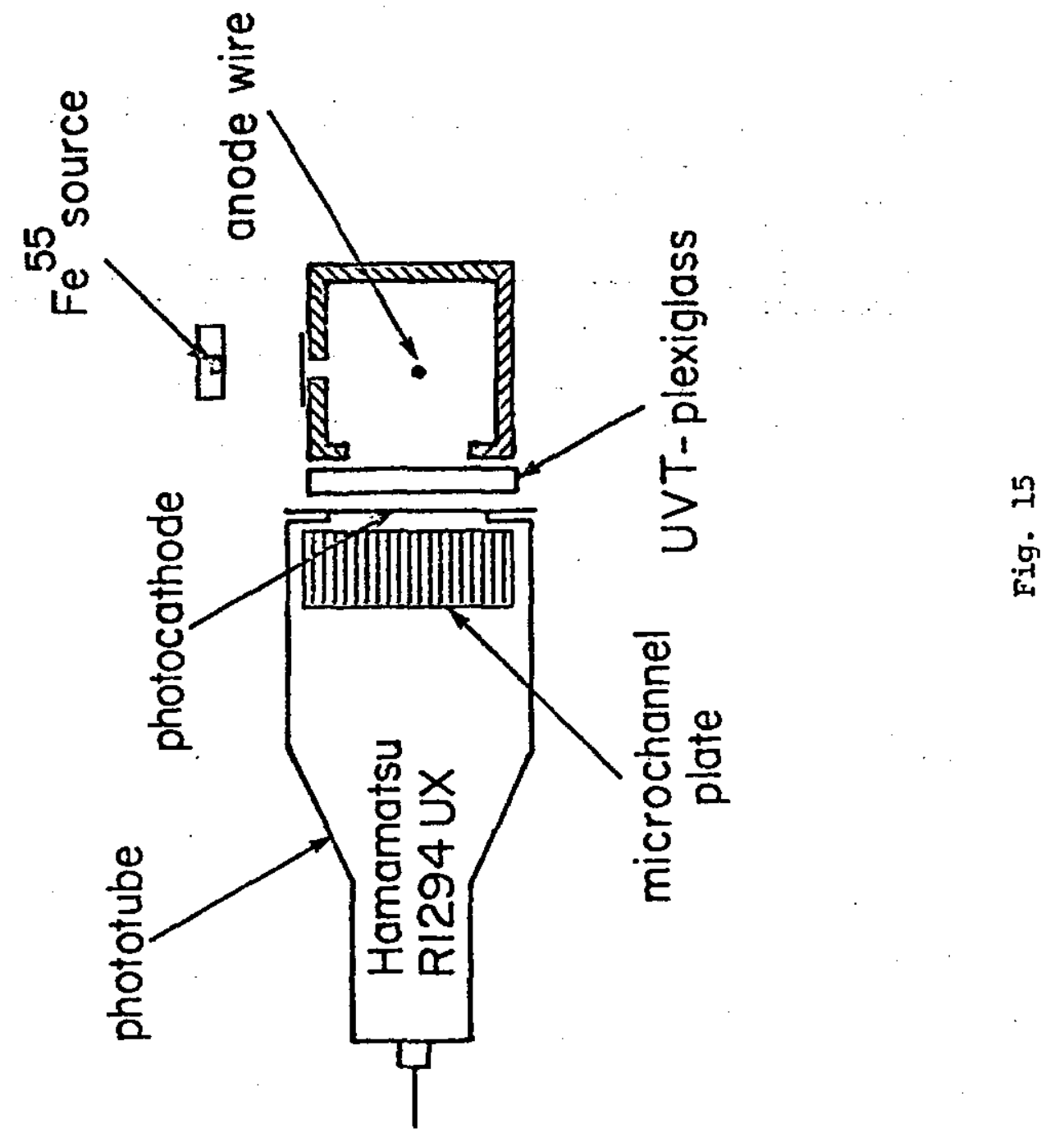




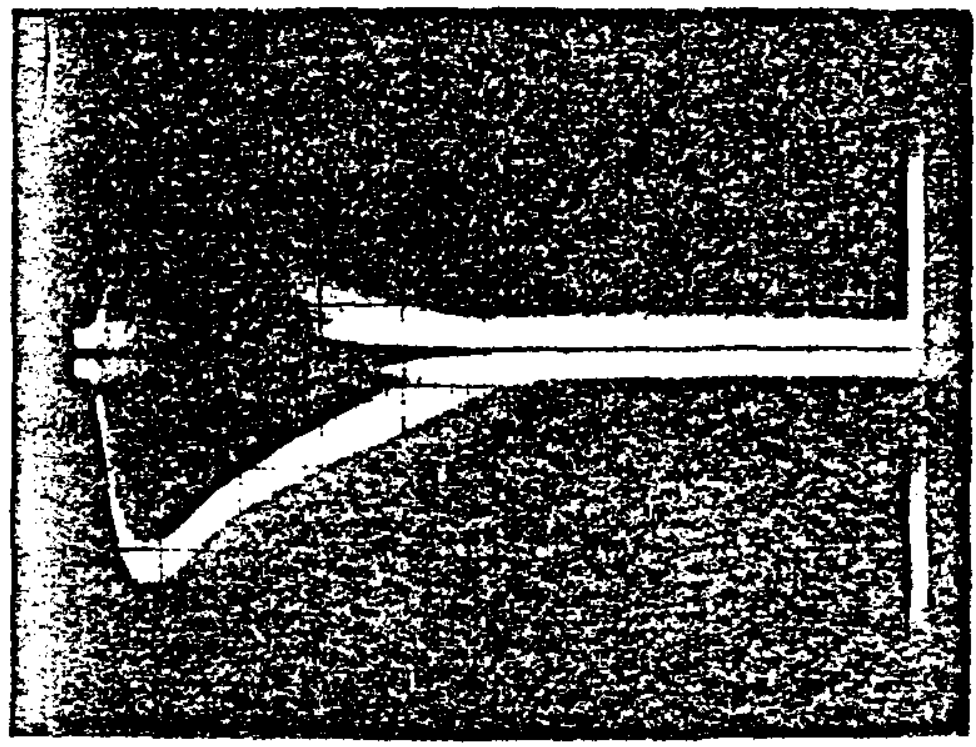

Fig. 16 


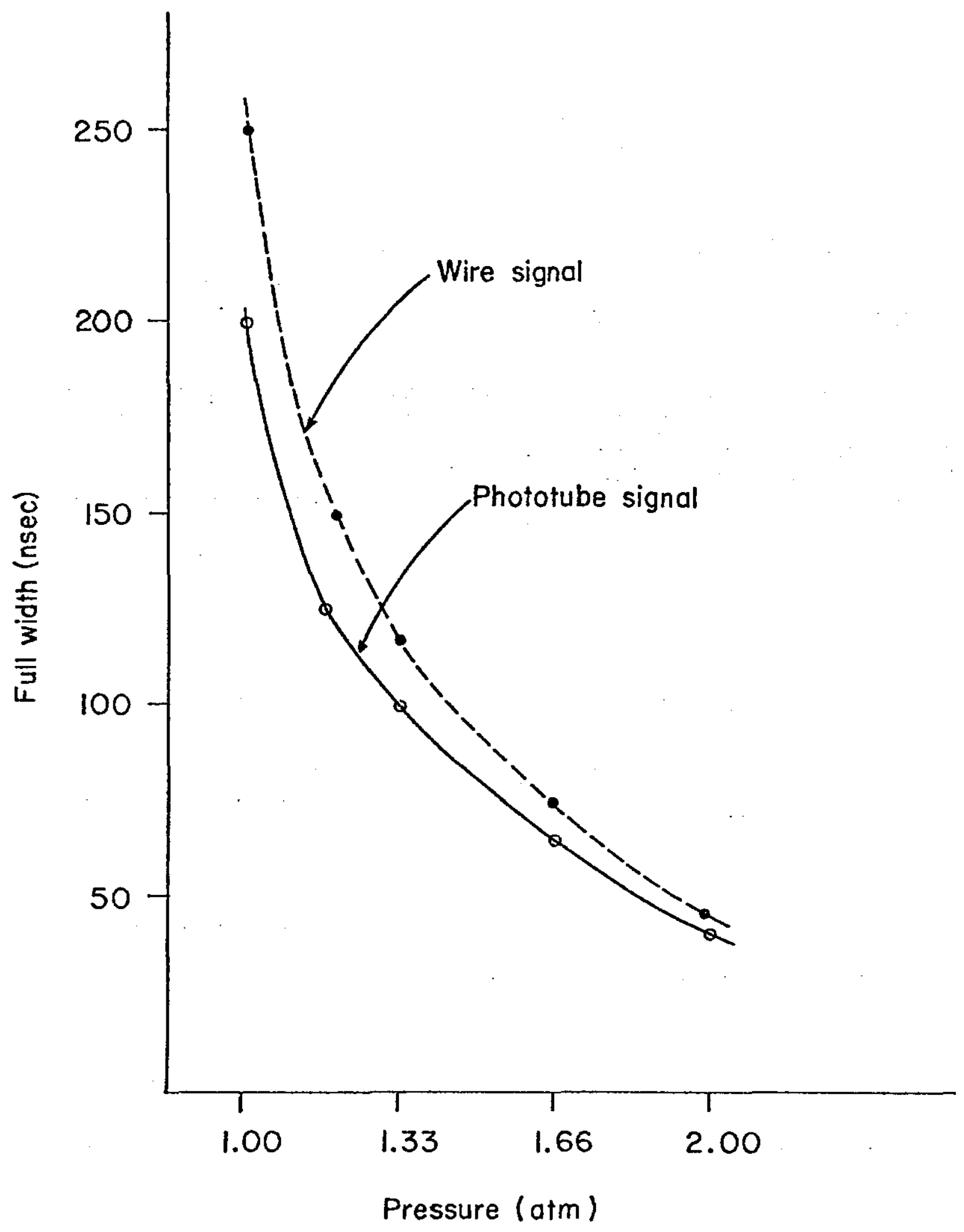

Fig. 17 


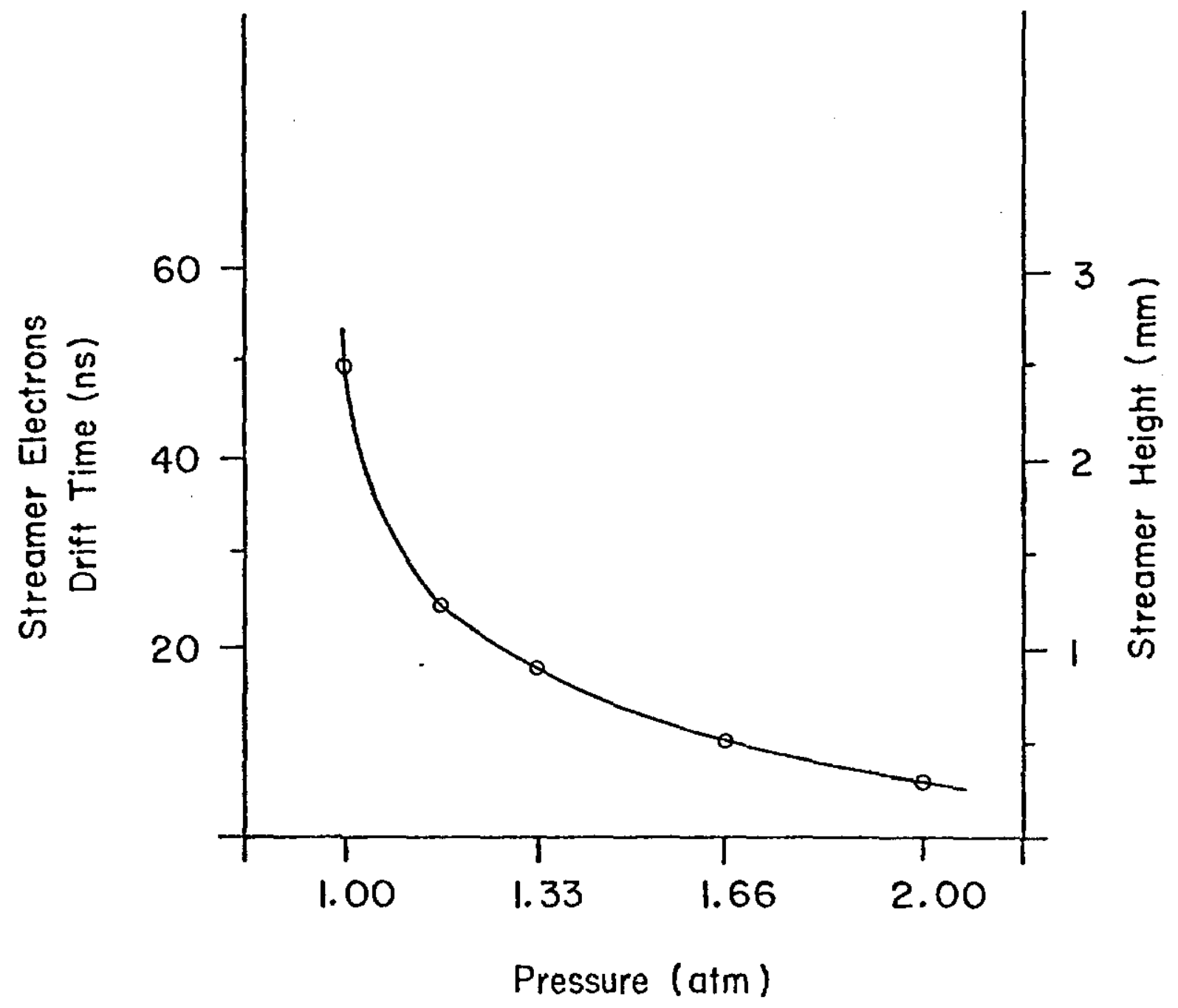

Fig. 18 